\title{
Macroinvertebrados acuáticos como indicadores biológicos de la calidad del agua en el Rio Gil González y tributarios más importantes, Rivas, Nicaragua
}

\author{
Salvatierra Suárez Thelma \\ Centro para la Investigación en Recursos Acuáticos de Nicaragua (CIRA/UNAN) \\ ENITEL Villa Fontana 400 metros al Norte. Teléfono: (505) 22786981-82 \\ thelma.salvatierra@cira-unan.edu.ni
}

\begin{abstract}
RESUMEN
Se estudio la fauna de los macoinvertebrados acuáticos como indicadores biológicos en ocho sitios en el Rio Gil González y tributarios más importantes, de los Municipios de Belén, Potosí y Buenos Aires, Rivas, Nicaragua. A través del análisis de la información producida en el CIRA/UNAN (Centro para la Investigación en Recursos Acuáticos de Nicaragua) a partir de dos muestreo (época seca y lluviosa) fueron utilizadas las variables de riqueza taxonómica, abundancia numérica e Índice Biological Monitoring Working Party (BMWP) modificado para Costa Rica, 2007. El objetivo del estudio fue determinar la calidad del agua en el Rio Gil González usando macroinvertebrados acuáticos como herramienta fundamental en el biomonitoreo hídrico. Fueron identificados 32 categorías taxonómicas en total, la Clase Insecta con más variedad de órdenes: Díptera, Ephemeroptera, Trichoptera y Coleóptera, siendo el Orden Díptera la categoría taxonómica con más variedad y frecuencia de Familias encontradas en todos los sitios de muestreo, con dos Familias dominantes Chironomidae y Cerapotogonidae. En general se encontró que la calidad ecológica (Índice BMWP'-CR) en los sitios analizados en el cauce principal y tributarios más importantes del Rio Gil González, fueron consideradas de buena calidad para los dos muestreos (Nov.2010 y Abril 2011), solamente en los sitios La Ermita, San Pedro y La Lagarta fueron determinadas aguas de regular calidad en el muestreo de Noviembre 2010.
\end{abstract}

Palabras claves: Macroinvertebrados, indicadores, calidad, tributarios, Nicaragua.

\section{INTRODUCCIÓN}

Los Macroinvertebrados Acuáticos (MIA) son los organismos que habitan en los sedimentos de los ecosistemas acuáticos, o en cualquier tipo de sustrato (hoja, tronco, macrofitas, entre otros). Se incluyen los individuos iguales o mayores a $250 \mu \mathrm{m}$, entre ellos se encuentran los turbelarios, nematodos, oligoquetos, hirudineos, insectos, arácnidos, crustáceos, gasterópodos y bivalvos. Algunos representantes pasan períodos de residencia en agua por tiempo cortos, otros prolongados y algunos exclusivamente acuáticos.

Son excelentes indicadores biológicos de las condiciones de calidad de un determinado recurso hídrico superficial. Cuando hay evidencias de contaminación orgánica o química los macroinvertebrados son utilizados para determinar la calidad del ecosistema acuático.

El conocimiento de los macroinvertebrados acuáticos en las zonas templadas ha sido ampliamente difundido por medio de los textos de Usinger (1956), Edmonson (1959), Hynes (1974), Merrit y Cummins (1996), Penak (1978), Wesenberg-Lund (1980) y McCafferty (1981); 
Roldan y Ramírez, 2008. Es así entonces que el uso de los macroinvertebrados acuáticos como indicadores biológicos para evaluar la calidad de las aguas es más frecuente en algunos países de Latinoamérica, en particular Colombia con la adecuación del índice Biological Monitoring Working Party (BMWP') modificado por Alba Tercedor y Sánchez Ortega, 1988, por el BMWP/Col por Roldan, 2003, por el BMWP/CR por Springer et al 2007; en México Weigel et al. (2002) desarrollaron un índice integrado para evaluar ríos en la zona centro occidental del país; en Chile Figueroa et al. (2003) y Leiva (2004) aplican el Índice Biótico de Familia de Hilsenhoff en varios ríos meridionales; Segnini, 2003. Para la región mesoamericana el IBFHilsenhoff (1987) fue adecuado para El Salvador (IBF-SV-2010), (Sermeño et al, 2010)

El Río Gil González y tributarios reviste especial importancia para los Municipios de Belén, Potosí y Buenos Aires del Departamento de Rivas, porque sus aguas son utilizadas principalmente para riego tanto en la parte alta como media y baja, así como también para recreación, lavado de ropa, baño, abrevadero de ganado, entre otras actividades, la sobre explotación y mal manejo del agua, por tal razón fueron propuestos los objetivos siguientes: determinar la composición de Familias, abundancia de los macroinvetebrados acuáticos y aplicar el Índice Biological Monitoring Working Party (BMWP/CR, 2007) para la evaluación de la calidad de las aguas en los ocho sitios analizados.

\section{MATERIALES Y METODOS}

El Río Gil González constituye el más extenso y caudaloso rio de los municipios de Belén, Potosí y Buenos Aires localizado entre las coordenadas 609 y 627 este y las 1272 y 1282 norte (North American Datum de 1927, NAD27), con un área total de $68.43 \mathrm{Km}^{2}$, y un perímetro aproximado de $56.41 \mathrm{~km}$.

Fueron seleccionados ocho sitios de muestreo, donde se recolectaron muestras de sedimento con material orgánico del fondo para el análisis cualitativo y cuantitativo de los macroinvertebrados acuáticos, en dos momentos Noviembre 2010 y Abril 2011. Se utilizo una red de captura de mano o de pantalla de 250 micras, en diferentes secciones del rio, la muestra es tamiza, depositada en recipientes de plástico y preservada con alcohol al 94\%. También fueron recolectados organismos en hojarasca. Las muestras fueron rotuladas con el nombre del sitio, fecha y hora de muestreo, posteriormente se colocaron en canastas de plástico de textura más fuerte y grandes con el propósito de conservarlas en buen estado hasta la llegada al laboratorio de Hidrobiología en el CIRA/UNAN.

Las muestras en el laboratorio fueron lavadas utilizando un tamiz de $250 \mu \mathrm{m}$ para separar los organismos de los sedimento, las muestras totalmente limpias fueron guardadas otra vez en recipientes de plástico $(250 \mathrm{ml})$ preservándolas con alcohol al $94 \%$ y rotuladas nuevamente con el nombre del lugar, fecha y hora de colecta.

Una vez que las muestras estuvieron listas para el análisis, el sedimento con material orgánico fue colocado en pocas cantidades en un plato petri revisando y separando los organismos uno por uno por medio de pinzas bajo un microscopio estereoscopio, seguidamente fueron colocadas 
en frascos de vidrio conteniendo alcohol y agregando 2-3 gotas de glicerina, para mantener blandas y flexibles las estructuras de los organismos. Cada muestra fue analizada de forma individual siguiendo los Procedimientos Operativos Normalizados (PON) descritos en el Manual de Calidad Analítica del Laboratorio de Hidrobiología.

Para la identificación del grupo de los macroinvertebrados acuáticos se utilizó un microscopio compuesto (Leica DM LS2) con objetivos de 4x, 10x, 25x, 40x y 100x provistos de un micrómetro ocular calibrado y microscopio estereoscópico Leica EZ4D, oculares de 10x fijos y cámara CMOS de 3,0 megapíxeles incorporada. Los individuos se clasificaron según Clase, Orden, Familia y Género (esta última cuando fue posible), utilizando las claves taxonómicas de Roldan (1988), Pennak (1978) y Merrit \& Cummins (1984). Fueron utilizadas ecuaciones matemáticas para determinar la densidad poblacional y abundancia de los individuos (Standard Methods, 2005).

Para determinar la calidad del agua en relación al aporte de materia orgánica en los sectores analizados, se utilizó el Índice Biological Monitoring Working Party (BMWP) modificado para Costa Rica. Este índice biótico establece la calidad de un determinado cuerpo de agua en relación al aporte de materia orgánica y la respuesta ecológica de las Familias encontradas de macroinvertebrados acuáticos.

\section{RESULTADOS}

\section{Composición taxonómica}

Para los dos muestreos en los ocho sitios analizados fueron identificados 32 categorías taxonómicas en total, distribuidas en dos grandes grupos Phyllum Arthropoda y Mollusca; dentro de los Artrópodos la dominancia fue dada por la Clase Insecta con más variedad de

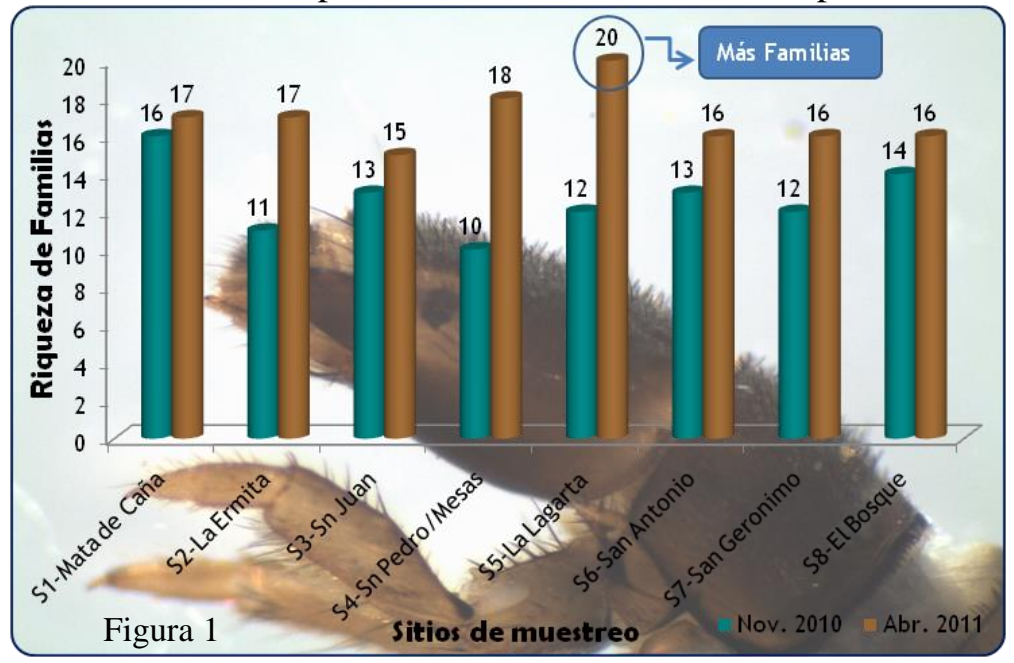
órdenes: Ephemeroptera, Odonata, Hemiptera, Coleoptera, Trichoptera y Diptera, siendo esta última categoría taxonómica con más variedad y frecuencia de Familias, con dos Familias dominantes Chironomidae y Cerapotogonidae.

En la Figura 1 se muestra el aporte de las categorías taxonómicas en los sitios analizados en el Rio Gil González en los dos muestreos, se observa que los aportes más altos fueron encontrados en el segundo muestreo (Abril 2011) y el sitio con más variedad de Familias fue en La Cruz o Lagarta con 20 Familias en el segundo muestreo (Época seca-Abril 2011), a diferencia del número de Familias encontradas para la época de lluvias (Nov.2010) que fue de 12 Familias en total. 


\section{Abundancia numérica}

Fueron encontrados en total 13201 individuos de macroinvertebrados

acuáticos, de los cuales 4256 fueron colectados en el primer muestreo (Nov. 2010) y 8945 en el segundo muestreo (Abril 2011). El número de individuos por sitio fue mayor en el segundo muestreo (Abril del 2011), así como en la contribución total, en la Figura 2 se

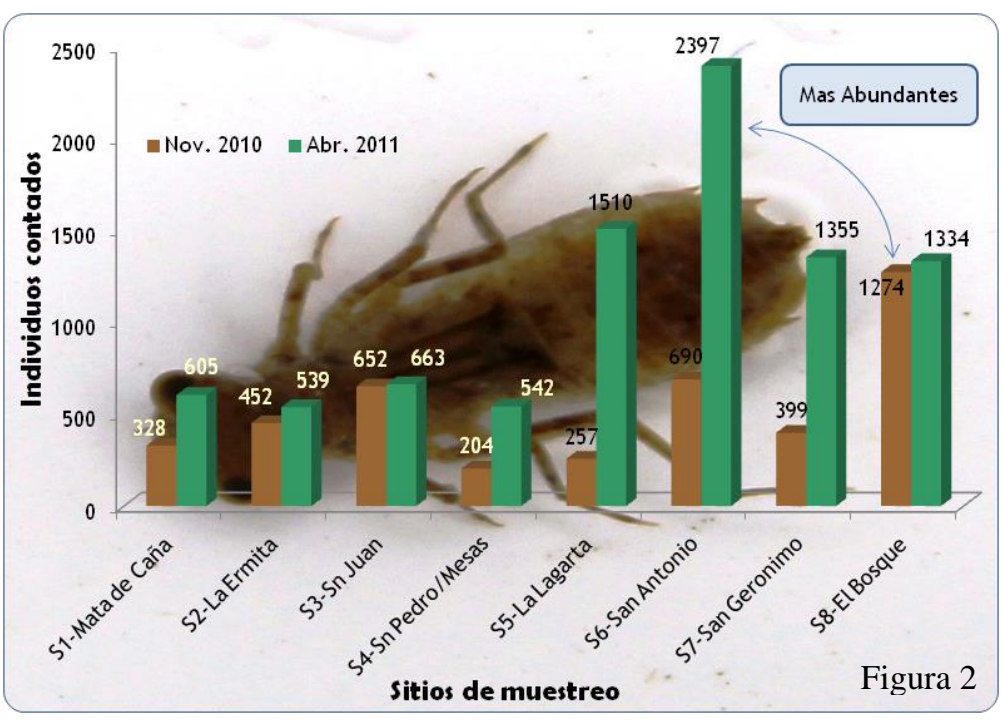
muestra el número de individuos encontrados para los dos muestreos, y se observa que las mayores abundancias fueron encontradas en los sitios: San Antonio, La Lagarta y San Gerónimo para la época seca (muestreo Abril 2011) y El Bosque en ambas épocas de muestreo.

\section{$\underline{\text { Estado de la calidad del agua a través del Índice Biótico (BMWP'-CR) }}$}

En la tabla siguiente se muestran los niveles de calidad de las aguas y los rangos de valores según el Índice BMWP'-CR (Springer, 2007) y el color para cartografía. En base a esta información se obtuvo los resultados para el Rio Gil González.

\begin{tabular}{|l|c|l|c|}
\hline Calidad & $\begin{array}{c}\text { Rangos de Valor } \\
\text { BMWP'-CR }\end{array}$ & \multicolumn{1}{|c|}{ Nivel de Calidad } & Color \\
\hline Excelente & $>120$ & Aguas de calidad excelente. & \\
\hline $\begin{array}{l}\text { Muy } \\
\text { Buena }\end{array}$ & $101-120$ & $\begin{array}{l}\text { Aguas de calidad buena, no contaminadas o no } \\
\text { alteradas de manera sensible. }\end{array}$ & \\
\hline Buena & $61-100$ & $\begin{array}{l}\text { Aguas de calidad regular, contaminación } \\
\text { moderada. }\end{array}$ & \\
\hline Regular & $36-60$ & Aguas de calidad mala, contaminadas. & \\
\hline Pobre & $16-35$ & Aguas de calidad mala, muy contaminadas. & \\
\hline $\begin{array}{l}\text { Muy } \\
\text { Crítica }\end{array}$ & $\leq 15$ & $\begin{array}{l}\text { Aguas de calidad muy mala, extremadamente } \\
\text { contaminadas. }\end{array}$ & \\
\hline
\end{tabular}

Para el primer muestreo (Noviembre 2010), los valores y la calidad según el índice BMWP'-CR las aguas de los sitios analizados en el Rio Gil González fueron consideradas de buena calidad, a excepción de los sitios La Ermita, San Pedro y La Lagarta fueron determinadas aguas de regular calidad. Estos resultados se corresponden con los valores de abundancia numérica analizadas en el primer muestreo que fue menor en estos sitios (Figura 2). En la Figura 3 se muestran los niveles de calidad obtenidos del Índice BMWP'-CR para el primer muestreo. 


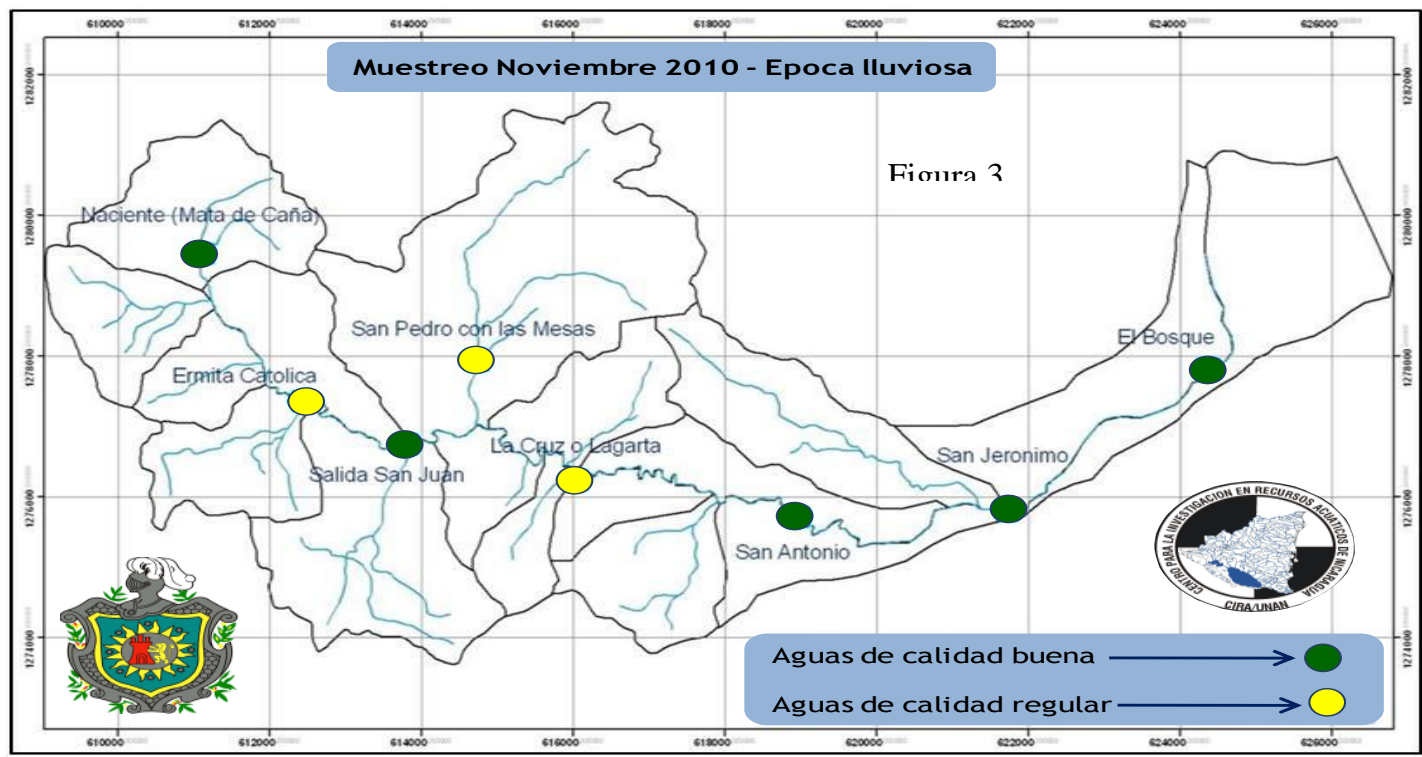

Para el segundo muestreo (Abril 2011), los valores y la calidad según el índice BMWP'-CR las aguas de todos los sitios analizados en el Rio Gil González fueron consideradas de buena calidad, estos resultados se corresponden con los valores de abundancia numérica analizadas en el segundo muestreo en los sitios analizados (Figura 2). En la Figura 4 se muestran los niveles de calidad obtenidos del Índice BMWP'-CR para el segundo muestreo.

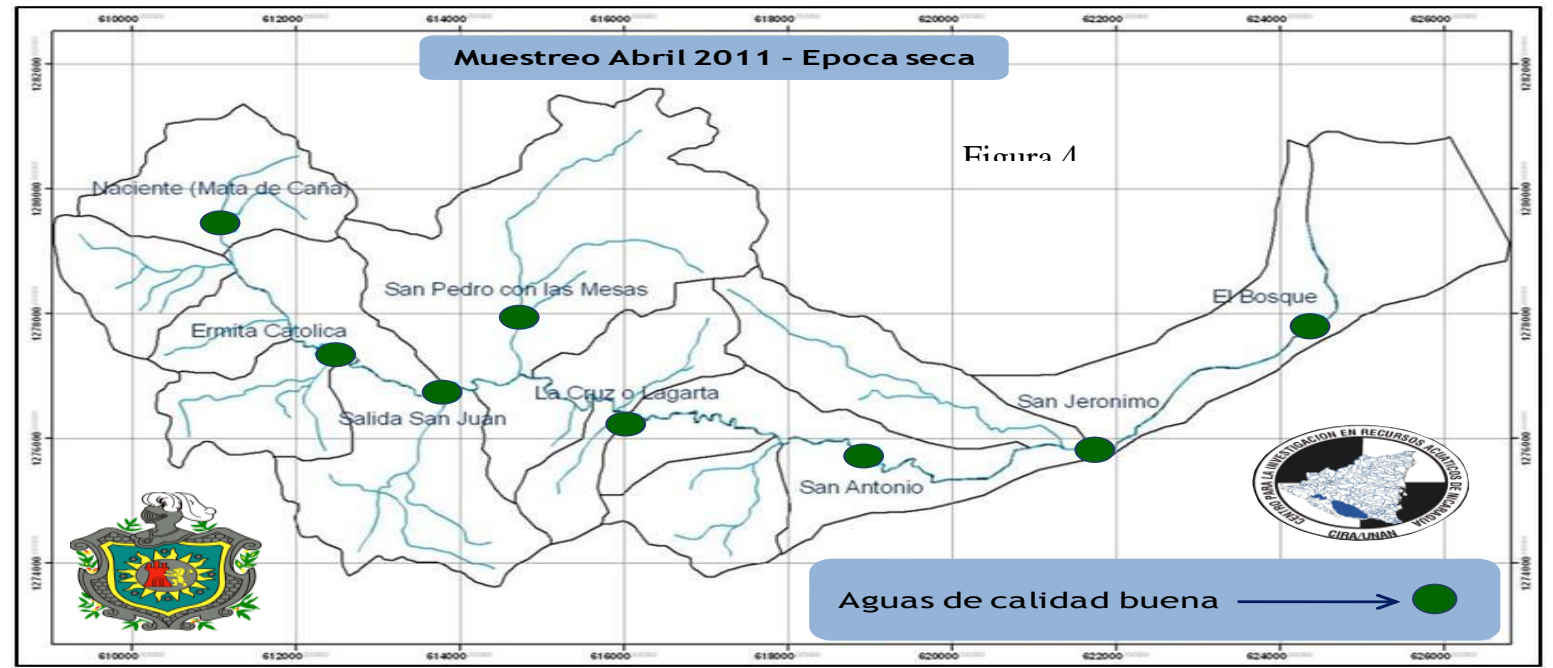

\section{DISCUSIÓN}

Los macroinvertebrados acuáticos (MIA) en los ocho sitios fue predominante por el Phyllum Arthropoda. Según Roldan y Ramírez (2008) el Phyllum Arthropoda representa el grupo más abundante de macroinvertebrados acuáticos. A este Phyllum pertenecen tres grandes grupos taxonómicos a saber: Crustacea, Insecta y Arachnida. En los sitios muestreados fueron encontrados los tres grupos mencionadas anteriormente, el grupo más dominante tanto en número de individuos como en categorías taxonómicas fue la Clase Insecta. 
El Subphyllum Crustacea estuvo representado por la Clase Ostracoda, según Roldan y Ramírez (2008) los crustáceos comprenden un grupo grande y diversificado en las aguas dulces. Según Springer, et al. 2010, aproximadamente un tercio de las especies de ostrácodas son dulceacuícolas. Son microcrustáceos (usualmente $<3 \mathrm{~mm}$ ) con un caparazón de dos valvas. La mayoría de las especies viven en el fondo del agua y muchas son filtradoras.

La clase Insecta fue la más dominante en número de individuos y categorías taxonómicas (6 órdenes y 29 familias). Los órdenes más diversos fueron Díptera con dos Familias dominantes Chironomidae y Cerapotogonidae; Ephemeroptera con dos Familias dominantes Leptohyphidae y Leptophlebiidae y Trichoptera con la Familia Hydropsychidae más abundante. El grupo de los insectos es ampliamente distribuido en el neotrópico y el más diverso en cuanto a las variedades de órdenes, familias, géneros y especies. Los resultados encontrados en este estudio con relación a la dominancia de los insectos es similar al de Figueroa et al (2003) en la cuenca del Río Damas, Chile, donde registró un total de 77 taxa (75\%) de los cuales correspondió a estados inmaduros de insectos. En los sitios seleccionados para este estudio los insectos fueron predominantes en particular los dípteros. Según Roldan y Ramírez (2008) los dípteros constituyen otro de los órdenes de insectos más complejos, abundantes y ampliamente distribuidos en el mundo. Según Springer, et al. 2010, los dípteros acuáticos habitan en mas tipos de agua que cualquier otro grupo de insectos, su biología es sumamente diversa y las larvas son muy variables en su morfología, aunque nunca poseen patas verdaderas (articuladas) en el tórax.

Dentro del grupo de la Clase Arachnida solamente los ácaros (subclase Acari) incluyen un gran número de especies acuáticas (Walter \& Proctor 1999, citado por Springer, et al. 2010). Dentro de este grupo las Hydrachnidiae (también conocidos como Hidracarina o Hydrachnida) es el grupo más diverso de ácaros dulceacuícolas y se encuentran en una gran diversidad de ambientes acuáticos (Di Sabatino et al. 2008, citado por Springer, et al. 2010). En los sitios analizados en el Rio Gil González fueron encontrados solamente 57 individuos contados en total en los sitios a excepción de La Ermita (S2) que no fue encontrado ningún individuo.

Es evidente que hay heterogeneidad con las Familias encontradas en el ecosistema acuático estudiado, esto muestra equilibrio ecológico y estabilidad en el sistema, conteniendo los sitios los elementos necesarios para mantener esa variedad, en particular con las familias de la Clase Insecta.

El Biological Monitoring Working Party (BMWP) fue creado en el año 1978 para evaluar los ríos de Gran Bretaña, posteriormente fue adaptado para la Península Ibérica en 1988, adecuado para los ríos del trópico por Gabriel Roldan en 2003, y modificado para Costa Rica en 2007.

Para el BMWP las familias poco tolerantes a la contaminación tienen registros con valores altos y las familias tolerantes tienen registros de valores bajos. El sistema BMWP, considera que un cuerpo de agua tiene un alto grado de contaminación cuando los valores obtenidos en el índice son bajos.

En el primer muestreo (Noviembre 2010), la calidad ecológica resultante en los sitios analizados en el Rio Gil González, se corresponde con el arrastre de material orgánico por las lluvias y no 
refleja el estado de la calidad ecológica en los sitios en mención; a diferencia del segundo muestreo (Abril 2011) la calidad de las aguas según el BMWP'-CR fue igual en todos los sitios (buena calidad), estos resultados tienen relación con las bajas velocidades de las corrientes y caudal base, que permite el establecimiento de diferentes poblaciones de macroinvertebrados acuáticos, en particular Familias del Orden Ephemeroptera y Trichoptera que se caracterizan por estar en aguas con buena provisión de oxigeno $\left(5.5-8.5 \mathrm{mg} . \mathrm{l}^{-1}\right)$, reflejando entones que en esta época es donde se establece mejor el estado de la calidad ecológica en los sitios en mención.

Con dos muestreos no es suficiente establecer la calidad definitiva del cuerpo de agua estudiado, con el análisis de la comunidad de los macroinvertebrados acuáticos en cuanto a composición taxonómica y abundancia numérica se puede determinar que el cauce principal y tributarios más importantes del Rio Gil González presentaron condiciones de buena calidad.

\section{CONCLUSIONES}

Se encontró dominancia del Phyllum Arthropoda, con la Clase Insecta la más dominante, con seis Ordenes Ephemeroptera, Odonata, Hemiptera, Coleoptera, Trichoptera y Díptera, siendo esta ultima la que presento más variedad de Familias.

Se encontró mayor dominancia y abundancia dentro de los Ordenes Ephemeroptera, Díptera y Trichoptera, siendo indicadores de aguas muy limpias a medianamente alteradas por material orgánico.

Fue encontrada la Familia Calamoceratidae solamente en el sitio ubicado en Mata de Caña, esta Familia es de excelente a muy buena calidad del agua según el índice BMWP'-CR a igual que la Familia Leptophlebiidae, la aparición de Calamoceratidae en Mata de Caña muestra condiciones favorables del mismo para el desarrollo de esta Familia.

Las categorías encontradas con el índice biótico (BMWP'-CR, Springer y colaboradores, 2007) reflejan aguas de buena calidad en la mayoría de los sitios analizados y de regular calidad solamente para los sitios La Ermita, San Pedro/Mesas y La Lagarta solamente en el muestreo de Noviembre 2010 (Época lluviosa).

El haber encontrado las categorías de calidad del agua de buena y regular con el índice biótico utilizado, no brinda una panorámica integral de la calidad del ecosistema acuático, al contrario solamente se relaciona con los aportes de materia orgánica y los cambios biológicos de los macroinvertebrados acuáticos bénticos a la contaminación orgánica.

La velocidad de la corriente y el caudal en la época de lluvias fueron variables hidrológicas que modificaron la variación de categorías taxonómicas y abundancia numérica de la comunidad de macroinvertebrados acuáticos en los cauces centrales y tributarios más importantes en el Rio Gil González. 


\section{RECOMENDACIONES}

Realizar cuatro muestreos para la colecta de macroinvertebrados acuáticos, dos en la época seca (Enero/Abril) y dos en la época de lluvias (Julio/Septiembre), para determinar la calidad de las aguas en los mismos sitios de muestreo analizados en este estudio para valorar con más precisión los indicadores biológicos, tomando en cuenta las variables físicas y químicas.

Seguir investigando la presencia de las Familia Calamoceratidae y Physidae en el sitio Mata de Caña, para conocer las variabilidades de estas dos familias.

\section{AGRADECIMIENTOS}

El estudio de los macroinvertebrados acuáticos como indicadores biológicos de la calidad del agua en el Rio Gil González y tributarios más importantes, es un componente del estudio evaluación sistemática de la calidad y cantidad del Rio Gil González y tributarios más importantes, componente del proyecto general Fortalecimiento de los gobiernos locales de Belén, Potosí y Buenos Aires para la gestión integrada de recursos hídricos de la subcuenca Gil González del Lago Cocibolca, con apoyo financiero de la Unión Europea y ejecutado por La Fundación Nicaragüense para el Desarrollo Sostenible (FUNDENIC SOS). Sin embargo los costos de análisis de las muestras, interpretación de los resultados y tiempo de la investigadora fueron asumidos en su totalidad por el Centro para la Investigación en Recursos Acuáticos de Nicaragua (CIRA/UNAN), como aporte científico técnico de extensión universitaria a las Alcaldías de los Municipios en mención.

Agradecimiento al Licenciado Rafael Varela integrante del Laboratorio de Hidrobiología del CIRA/UNAN, por su valiosa participación tanto en las salidas de campo como en el análisis cualitativo y cuantitativo de los MIA.

\section{BIBLIOGRAFÍA}

Figueroa, R., Valdovinos, C., Araya, E. y Parra., O., 2003. Macroinvertebrados bentónicos como indicadores de calidad de agua de ríos del sur de Chile. Unidad de Sistemas Acuáticos, Centro de Ciencias Ambientales EULA-Chile, Universidad de Concepción, Casilla 160-C, Concepción, Chile. Revista Chilena de Historia Natural.

Hilsenhoff, W.L., 1987. An improved biotic index of organic stream pollution. The great lakes entomologist J.N. Benthol. Vol. 20. No.1

Merrit, R. \& Cummins, K., 1984. An introduction to the aquatic insects of North America. Second Edition. Kundall / Hunt Publishing Company, Iowa. 711 p.

Pennak, W. Robert. 1978. Fresh Water Invertebrates of the United States. 803 pag

Roldan, G., 1998. Guía para el estudio de los macroinvertebrados acuáticos del Departamento de Antioquia. Universidad de Antioquia, Colombia. 
Roldan, P. G. y Ramírez, R. J., 2008. Fundamentos de limnología neotropical. 2a.ed.Medellin, Editorial Universidad de Antioquia. 442 p.

Samuel Segnini, 2003. El uso de los macroinvertebrados bentónicos como indicadores de la condición ecológica de los cuerpos de agua corriente. ECOTROPICOS 16(2):45-63 2003 Sociedad Venezolana de Ecología. Universidad de Los Andes. Facultad de Ciencias. Departamento de Biología. Laboratorio de Ecología de Insectos. La Hechicera. Mérida. Venezuela. E-mail: segninis@ula.ve

Sermeño, J.M., Serrano, L., Springer, M., Paniagua, M, R., Perez, D., Rivas, A,W., Menjivar, R, A., Bonilla, B, L., Carranza, F, A., Flores, J, M., Gonzales, C., Gutierrez, P,E., Monterrosa, A, J,. y Arias, A, Y., 2010. Determinación de la calidad ambiental de las aguas de los ríos de El Salvador, utilizando invertebrados acuáticos: índice biológico a nivel de familias de invertebrados acuáticos en El Salvador (IBF-SV-2010). 1ª. Ed.-San Salvador, El Salvador. Editorial Universitaria (UES). Numero de paginas 39.

Springer, M., Vasquez, D., Castro A, y Kohlmann, B., 2007. Uso del Índice BMWP'-CR de la calidad del agua. Universidad de Costa Rica.

Springer, M., Ramirez, A. y Hanson, P. 2010 (Ed). Revista de Biología Tropical. Macroinvertebrados de agua dulce de Costa Rica I. San Jose Costa Rica-Volumen 58 (Supl.4). ISSN-0034-7744. Págs. 240

Standard Methods for the Examination of water and wastewater 21 th Edition. Association. Washington. 2005 (Sección 10500: 10-63 al 10-78 pag). 